\title{
A RECURRENCE RELATION GENERALIZING THOSE OF APÉRY
}

\author{
RICHARD ASKEY and J. A. WILSON
}

(Received 4 November 1982)

Communicated by J. H. Loxton

\section{Abstract}

A three term recurrence relation is found for

$$
g_{n}=\sum_{k=0}^{n}\left(\begin{array}{l}
n \\
k
\end{array}\right)\left(\begin{array}{c}
n+a+d \\
k+d
\end{array}\right)\left(\begin{array}{c}
n+k+b+e \\
k+e
\end{array}\right)\left(\begin{array}{c}
n+k+c+f \\
k+f
\end{array}\right)
$$

when $a+d=b+c$. This includes the recurrence relations of Apery associated with $\zeta(3), \zeta(2)$ and $\log 2$ as special or limiting cases.

1980 Mathematics subject classification (Amer. Math. Soc.): 33 A 30, 10 A 35.

In the fall of $1978 \mathrm{~A}$. van der Poorten gave lectures on Apéry's proof of the irrationality of $\zeta(3)$ at a number of North American universities. The lecture was delightful. An approximation can be made by reading his published version of the lecture [14], but the lecture was even more exciting. See Mendes-France [6] for some comments similar to those van der Poorten left out of his published version. As a result of these lectures many people became aware that Apéry had shown that

$$
b_{n}=\sum_{k=0}^{n}\left(\begin{array}{l}
n \\
k
\end{array}\right)^{2}\left(\begin{array}{c}
n+k \\
k
\end{array}\right)^{2}
$$

satisfies the three term recurrence relation

$$
n^{3} b_{n}+(n-1)^{3} b_{n-2}=\left(34 n^{3}-51 n^{2}+27 n-5\right) b_{n-1}
$$


and that

$$
c_{n}=\sum_{k=0}^{n}\left(\begin{array}{l}
n \\
k
\end{array}\right)^{2}\left(\begin{array}{c}
n+k \\
k
\end{array}\right)
$$

satisfies

$$
n^{2} c_{n}-(n-1)^{2} c_{n-2}=\left(11 n^{2}-11 n+3\right) c_{n-1} .
$$

The existence of one recurrence relation of this type could be an accident but the existence of two suggested to a number of people that there should be more. We received a few letters asking about the existence of three term recurrence relations for a number of sequences. The most common example was

$$
d_{n}=\sum_{k=0}^{n}\left(\begin{array}{l}
n \\
k
\end{array}\right)\left(\begin{array}{c}
n+k \\
k
\end{array}\right)^{2}
$$

It is easy to see that $d_{n}$ satisfies a four term recurrence relation, and that

$$
e_{n}=\sum_{k=0}^{n}\left(\begin{array}{l}
n \\
k
\end{array}\right)^{3}\left(\begin{array}{c}
n+k \\
k
\end{array}\right)
$$

satisfies a five term one. It is also very likely that they do not satisfy a linear recurrence relation of a lower order. This was not clear to the people we wrote to, and to be honest it is not clear when the sequences are written in this form. Fortunately, there is a much better way to write them, and in this other form it is clear that (5) is a little more complicated than (3) and that (6) is much more complicated than (1). The other form of writing these series is old, and has been studied by Euler, Pfaff, Gauss, Kummer, Jacobi and many others, so it should not be as unknown as it is. It is called hypergeometric series.

A generalized hypergeometric series, or better, a hypergeometric series, is a series $\sum c_{k}$ with $c_{k+1} / c_{k}$ a rational function of $k$. This rational function is usually factored, so

$$
\frac{c_{k+1}}{c_{k}}=\frac{\left(k+a_{1}\right) \cdots\left(k+a_{p}\right) x}{\left(k+b_{1}\right) \cdots\left(k+b_{q}\right)(k+1)},
$$

and if $c_{0}=1$ this gives

$$
{ }_{p} F_{q}\left(\begin{array}{l}
a_{1}, \ldots, a_{p} \\
b_{1}, \ldots, b_{q}
\end{array} ; x\right):=\sum_{k=0}^{\infty} \frac{\left(a_{1}\right)_{k} \cdots\left(a_{p}\right)_{k} x^{k}}{\left(b_{1}\right)_{k} \cdots\left(b_{q}\right)_{k} k !} .
$$

The shifted factorial $(a)_{k}$ is defined by

$$
(a)_{k}:=a(a+1) \cdots(a+k-1)=\Gamma(a+k) / \Gamma(a) .
$$

Gauss [4], defined two ${ }_{2} F_{1}$ series to be contiguous if there is a change of 1 in one of the parameters and the others do not change. If $F$ denotes ${ }_{2} F_{1}(a, b ; c ; x)$ then $F(a+)$ will denote ${ }_{2} F_{1}(a+1, b ; c, x)$, and obvious generalizations of this notation will be used in the following. Gauss showed that a ${ }_{2} F_{1}$ and any two series 
that are contiguous to it are linearly related, and explicitly gave the $\left(\begin{array}{l}6 \\ 2\end{array}\right)=15$ identities. Since ${ }_{2} F_{1}(a, b ; c ; x)$ is symmetric in $a$ and $b$ there are really nine different identities. Gauss took one of his identities, set $x=1$, and it reduced to a two term recurrence relation. He iterated this and used it to prove that

$$
{ }_{2} F_{1}(\underset{c}{a, b} ; 1)=\frac{\Gamma(c) \Gamma(c-a-b)}{\Gamma(c-a) \Gamma(c-b)} .
$$

The Gauss contiguous relations can be iterated, so $_{2} F_{1}(a, b ; c ; x)$ and any two ${ }_{2} F_{1}$ 's whose parameters differ by integers from those of this ${ }_{2} F_{1}$ are linearly related. In particular if

$$
p_{n}={ }_{2} F_{1}(-n, n+a+b+1 ; a+1 ; t)
$$

then

$$
a_{n} p_{n+1}+b_{n} p_{n}+c_{n} p_{n-1}=0, \quad n=1,2, \ldots,
$$

for some $a_{n}, b_{n}, c_{n}$, and they can be found explicitly. The above statements can be made much stronger, and bounds on the degree of the coefficients in any of the parameters can be given. Without this the results are obvious, since we could take $c_{n}=0, b_{n}=-p_{n+1}, a_{n}=p_{n}$, which is a triviality. For the fundamental relations of Gauss the coefficients are linear in each parameter and in the variable $x$.

Since

$$
\frac{d}{d x}{ }_{2} F_{1}(a, b ; c, x)=\frac{a b}{c}{ }_{2} F_{1}(a+1, b+1 ; c+1 ; x)
$$

the relation connecting ${ }_{2} F_{1}(a, b ; c, x),{ }_{2} F_{1}(a+1, b+1 ; c+1 ; x)$ and ${ }_{2} F_{1}(a+2, b+2 ; c+2 ; x)$ can be rewritten as a second order differential equation. It is well known that ${ }_{p} F_{q}\left(a_{1}, \ldots, a_{p} ; b_{1}, \ldots, b_{q} ; x\right)$ satisfies a differential equation of order $q+1$ when $p \leqslant q+1$. The condition $p \leqslant q+1$ is necessary to obtain convergence of the series (8). It is not quite as well known that such a function and $q+1$ of them that are contiguous to this function are linearly related. See Rainville [8, Chapter 14]. Kummer [5] considered both ${ }_{2} F_{1}$ and ${ }_{1} F_{1}$ in detail, and at the end of the second part of this paper he wrote that he had tried to study

$$
{ }_{3} F_{2}(a, b, c ; d, e ; x)
$$

but only when $x=1$ had he been able to do much. He only stated one transformation formula, but linear three term relations exist between a ${ }_{3} F_{2}$ and any two series contiguous to it when the power series variable is one. Again these can be iterated, and a few of these relations have been recorded in print. The complete listing of these three term relations was only published in the late 1970's. One list is in Wilson [17], a second listing in very compact form is in Raynal [10].

At the ${ }_{4} F_{3}$ level a function and any four functions contiguous to it are linearly related. When $x=1$ it is usually (or always, we have not checked thoroughly) 
true that $\mathrm{a}_{4} F_{3}$ and three functions contiguous to it are linearly related. To find cases when a function and two contiguous series are linearly related we ask, by analogy with the ${ }_{3} F_{2},{ }_{2} F_{1}$ cases, if there are any ${ }_{3} F_{2}$ 's that can be summed. Being able to sum a series that has a free parameter, or more, is usually an indication that a two term recurrence relation exists between contiguous series in this family, and what one hopes is that when new parameters are added the order of the recurrence or contiguous relations will not increase very much. Pfaff [7] summed the following series:

$$
{ }_{3} F_{2}\left(\begin{array}{c}
-n, a, b \\
c, a+b+1-n-c
\end{array} ; 1\right)=\frac{(c-a)_{n}(c-b)_{n}}{(c)_{n}(c-a-b)_{n}} .
$$

This series was evaluated again by Saalschütz, almost one hundred years after Pfaff, and since Pfaff's work was not well known this identity was known as Saalschütz's until recently. The condition the parameters satisfy is

$$
-n+a+b+1=c+a+b+1-n-c,
$$

or

$$
1+\sum a_{i}=\sum b_{i}
$$

If $p=q+1$, if the series terminates, that is, if one of the $a_{i}$ 's is a negative integer, if the power variable is one, and if (12) holds the series is called balanced. The above analogy suggests that a balanced ${ }_{4} F_{3}$ and any two series contiguous to it are likely related. This is not true, since a series contiguous to a balanced series is not balanced. For the sum of the top parameters will be equal to the sum of the bottom ones, or will be two more than the sum of the bottom ones. This suggests redefining contiguous for balanced series. Change two parameters by one each in such a way that the new series is still balanced. To see if this is reasonable, observe that $b_{n}$ defined in (1) can be written as

$$
b_{n}={ }_{4} F_{3}\left(\begin{array}{c}
-n,-n, n+1, n+1 ; 1) . \\
1,1,1
\end{array}\right.
$$

This is a balanced ${ }_{4} F_{3}$, as is $b_{n+1}$ and $b_{n-1} \cdot c_{n}$ from (3) is

$$
c_{n}={ }_{3} F_{2}\left(\begin{array}{c}
-n,-n, n+1 ; 1 \\
1,1
\end{array}\right)
$$

and so is a ${ }_{3} F_{2}$ with power series variable equal to one. Thus both should satisfy three term recurrence relations. The other two sequences

$$
d_{n}={ }_{3} F_{2}\left(\begin{array}{c}
-n, n+1, n+1 ;-1 \\
1,1
\end{array}\right)
$$

and

$$
e_{n}={ }_{4} F_{3}\left(\begin{array}{c}
-n,-n,-n, n+1 ;-1 \\
1,1,1
\end{array}\right)
$$


do not satisfy the right type of restrictions. $d_{n}$ satisfies a four term recurrence relation, and $e_{n}$ satisfies a five term recurrence relation. One gets a three term relation for

$$
\sum_{k=0}^{n}\left(\begin{array}{l}
n \\
k
\end{array}\right)\left(\begin{array}{c}
n+k \\
k
\end{array}\right)^{2}(-1)^{k}={ }_{3} F_{2}\left(\begin{array}{c}
-n, n+1, n+1 ; 1 \\
1,1,1
\end{array}\right)
$$

The heuristic argument given above leads to the right conclusion. A balanced ${ }_{4} F_{3}$ and any two balanced contiguous series to it are linearly related. These relations were first treated in detail by the same two who listed the ${ }_{3} F_{2}$ relations. Wilson [18] gave a complete list, and Raynal [11] gave a method for deriving them and stated the fundamental ones. Wilson's list is more convenient to use, but it takes more space to print.

To see how to generalize (1) and (2) consider

$$
g_{n}=\sum_{k=0}^{n}\left(\begin{array}{l}
n \\
k
\end{array}\right)\left(\begin{array}{c}
n+a+d \\
k+d
\end{array}\right)\left(\begin{array}{c}
n+k+b+e \\
k+e
\end{array}\right)\left(\begin{array}{c}
n+k+c+f \\
k+f
\end{array}\right) .
$$

We do not assume the parameters are integers, and define

$$
\left(\begin{array}{l}
a \\
b
\end{array}\right)=\frac{a !}{(a-b) ! b !}=\frac{\Gamma(a+1)}{\Gamma(a-b+1) \Gamma(b+1)}
$$

if $a$ is not a negative integer. If $a$ is a negative integer then the sum (19) can still make sense if there is another gamma function that is in the denominator and each pole of $\Gamma(x)$ is cancelled by a zero of $[\Gamma(y)]^{-1}$. One has to be careful in taking limits, but this can usually be done. To avoid these problems assume they do not arise. The series for $g_{n}$ can be rewritten as

$$
\begin{aligned}
g_{n}= & \left(\begin{array}{c}
n+a+d \\
d
\end{array}\right)\left(\begin{array}{c}
n+b+e \\
e
\end{array}\right)\left(\begin{array}{c}
n+c+f \\
f
\end{array}\right) \\
& \cdot{ }_{4} F_{3}\left(\begin{array}{c}
-n,-n-a, n+b+e+1, n+c+f+1 \\
d+1, e+1, f+1
\end{array} ; 1\right) .
\end{aligned}
$$

This ${ }_{4} F_{3}$ is balanced when $b+c-a=d$ or $a+d=b+c$. Without a translation it would be hard (or impossible) to see why this is a natural condition to assume.

The series in (21) will be denoted by

$$
F_{n}={ }_{4} F_{3}\left(\begin{array}{c}
-n,-n-a, n+b+e+1, n+c+f+1 \\
d+1, e+1, f+1
\end{array} ; .\right.
$$

If we show that

$$
A_{n} F_{n+1}+B_{n} F_{n}+C_{n} F_{n-1}=0
$$

then

$$
A_{n}^{\prime} g_{n+1}+B_{n}^{\prime} g_{n}+C_{n}^{\prime} g_{n-1}=0
$$


with

$$
\begin{aligned}
& A_{n}^{\prime}=A_{n} \frac{(n+a+1)(n+b+1)(n+c+1)}{(n+a+d+1)(n+b+e+1)(n+c+f+1)}, \\
& B_{n}^{\prime}=B_{n}, \\
& C_{n}^{\prime}=C_{n} \frac{(n+a+d)(n+b+e)(n+c+f)}{(n+a)(n+b)(n+c)} .
\end{aligned}
$$

Note that $F_{n}, F_{n-1}$ and $F_{n+1}$ are balanced series related in the same way as are the series $F, F\left(a+, b+, c_{-}, d_{-}\right)$, and $F(a-, b-, c+, d+)$, where $F$ is a balanced series

$$
F={ }_{4} F_{3}\left(\begin{array}{c}
a, b, c, d \\
e, f, g
\end{array} ; 1\right)
$$

Note also that $F(a+, c-)$ is a balanced series contiguous to $F$ and $F(a+$, $\left.b+, c_{-}, d_{-}\right)$and that $F(b-, d+)$ is contiguous to $F$ and $F(a-, b-, c+, d+)$. The contiguous relation

$$
\begin{aligned}
& \frac{(e-b)(f-b)(g-b)}{c(a-b+1)}[F(a+, b-)-F] \\
& -\frac{(e-d)(f-d)(g-d)}{a(c-d+1)}[F(c+, d-)-F]+(d-b) F=0
\end{aligned}
$$

from [18] gives a relation connecting $F, F\left(a+, c_{-}\right)$, and $F(b-, d+)$, another relation connecting $F, F(a+, b-)$, and $F\left(a+, b+, c_{-}, d_{-}\right)$, and a third connecting $F, F(b-, d+)$, and $F(a-, b-, c+, d+)$. Eliminating $F\left(a+, c_{-}\right)$and $F(b-, d+)$ from these three gives the desired three term relation. In terms of $F_{n}$ it is formula (23) with

$$
\begin{aligned}
& A_{n}=(n+d+1)(n+e+1)(n+f+1)(n+a+d+1)(n+a+e+1) \\
& \cdot(n+a+f+1)(n+b+e+1)(n+c+f+1) / D_{1}, \\
& C_{n}=(n+c+f-d)(n+c+f-e)(n+c)(n+b+e-d)(n+b) \\
& \quad \cdot(n+b+e-f) n(n+a) / D_{2}, \\
& B_{n}=-(n+a+d+1)(n+a+e+1)(n+a+f+1)(n+b+e+1) \\
& \quad \cdot(n+c+f+1)[(n+d+1)(n+e+1)(n+f+1) \\
&\quad+(n+a+1)(2 n+b+e+2)(2 n+a+c+f+2)] \cdot D_{1}^{-1} \\
&-(n+b+e-d)(n+b)(n+b+e-f)(n+a) n \\
& \quad \cdot[(n+c+f-d)(n+c+f-e)(n+c) \\
& \quad-(2 n+a+b+e+1) n(n+c+f+1),
\end{aligned}
$$




$$
\begin{aligned}
D_{1}= & (n+d+1)(n+e+1)(n+f+1)(n+b+e+1)(2 n+a+c+f+2) \\
+ & (n+c+f-d+1)(n+c+f-e+1)(n+c+1)(n+a+1) \\
& \cdot(2 n+b+e+2)+(2 n+c+f+2)(n+a+1)(2 n+b+e+2) \\
& \cdot(n+b+e+1)(2 n+a+c+f+2),
\end{aligned}
$$

and

$$
\begin{aligned}
D_{2}= & (n+d)(n+e)(n+f)(n+b+e)(2 n+a+c+f) \\
& +(n+c+f-d)(n+c+f-\cdot e)(n+c)(n+a)(2 n+b+e) \\
& +(2 n+c+f)(n+a)(2 n+b+e)(n+b+e)(2 n+a+c+f) .
\end{aligned}
$$

To find a recurrence relation for $c_{n}$, and more generally for

$$
\begin{aligned}
h_{n} & =\sum_{k=0}^{n}\left(\begin{array}{l}
n \\
k
\end{array}\right)\left(\begin{array}{c}
n+a+d \\
k+d
\end{array}\right)\left(\begin{array}{c}
n+k+b+e \\
k+b
\end{array}\right) \\
& =\left(\begin{array}{c}
n+a+d \\
d
\end{array}\right)\left(\begin{array}{c}
n+b+e \\
e
\end{array}\right)_{3} F_{2}\left(\begin{array}{c}
-n,-n-a, n+b+e+1 \\
d+1, e+1
\end{array} ; 1\right)
\end{aligned}
$$

divide (23) by $f$ and let $f \rightarrow \infty$. This gives a recurrence relation for the ${ }_{3} F_{2}$ in (26), and so also for $h_{n}$. When this recurrence relation is written there is a $c$ in it, but not in the ${ }_{3} F_{2}$. Remove the $c$ by using $a+d=b+c$.

If $d \rightarrow \infty$ in (22) with $a+d=b+c$ and $b$ and $c$ fixed, the resulting series is

$$
\begin{aligned}
&{ }_{3} F_{2}\left(\begin{array}{c}
-n, n+b+e+1, n+c+f+1 \\
e+1, f+1
\end{array}\right) \\
&=\left[\left(\begin{array}{c}
n+b+e \\
e
\end{array}\right)\left(\begin{array}{c}
b+c+f \\
f
\end{array}\right)\right]^{-1} \\
& \cdot \sum_{k=0}^{n}\left(\begin{array}{l}
n \\
k
\end{array}\right)\left(\begin{array}{c}
n+k+b+e \\
k+e
\end{array}\right)\left(\begin{array}{c}
n+k+c+f \\
k+f
\end{array}\right)(-1)^{k} .
\end{aligned}
$$

This gives a three term recurrence relation for the series defined in (17).

Similarly one can find a three term recurrence relation satisfied by

$$
\sum_{k=0}^{n}\left(\begin{array}{l}
n \\
k
\end{array}\right)^{3}\left(\begin{array}{c}
3 n+k+1 \\
k
\end{array}\right)(-1)^{k}={ }_{4} F_{3}\left(\begin{array}{c}
-n,-n,-n, 3 n+2 ; 1 \\
1,1,1
\end{array}\right)
$$

and many other sequences which may have some arithmetic significance.

This is not the end of the three term recurrence relations. Van der Poorten [14] mentioned that $\mathrm{T}$. Cusick had found two more.

$$
u_{n}=\sum_{k=0}^{n}\left(\begin{array}{l}
n \\
k
\end{array}\right)^{4}
$$


satisfies

$$
n^{3} u_{n}=2(2 n-1)\left(3 n^{2}-3 n+1\right) u_{n-1}+(4 n-3)(4 n-4)(4 n-5) u_{n-2}
$$

and

$$
v_{n}=\sum_{k=0}^{n}\left(\begin{array}{l}
n \\
k
\end{array}\right)^{3}
$$

satisfies

$$
n^{2} v_{n}=\left(7 n^{2}-7 n+2\right) v_{n-1}+8(n-1)^{2} v_{n-2} .
$$

These do not fit directly into the above pattern, since

$$
u_{n}={ }_{4} F_{3}\left(\begin{array}{l}
-n,-n,-n,-n \\
1,1,1
\end{array}\right.
$$

is not balanced and

$$
v_{n}={ }_{3} F_{2}\left(\begin{array}{c}
-n,-n,-n \\
1,1
\end{array} ;-1\right)
$$

has the wrong power series variable.

However both series can be transformed to the appropriate type of series. To transform (32) recall a transformation of Whipple [16]

$$
\begin{aligned}
& { }_{3} F_{2}\left(\begin{array}{c}
a, b, c \\
a+1-b, a+1-c
\end{array} ; x\right) \\
& =(1-x)^{-a}{ }_{3} F_{2}\left(\begin{array}{c}
a+1-b-c, a / 2,(a+1) / 2 \\
a+1-b, a+1-c
\end{array} ; \frac{-4 x}{(1-x)^{2}}\right) .
\end{aligned}
$$

When $x=-1$ this gives

$$
{ }_{3} F_{2}\left(\begin{array}{c}
a, b, c \\
a+1-b, a+1-c
\end{array} ;-1\right)=2_{3}^{-a} F_{2}\left(\begin{array}{c}
a+1-b-c, a / 2,(a+1) / 2 ; 1 \\
a+1-b, a+1-c
\end{array}\right) .
$$

The series on the left is said to be well poised, since the parameters can be paired, top ones with bottom ones, so that the sum is constant. The $n$ ! in the denominator is considered as $(1)_{n}$. A well poised ${ }_{3} F_{2}$ at $x=-1$ can be transformed to $a_{3} F_{2}$ at $x=1$; and series whose parameters differ by integers correspond on the two sides. Thus Cusick's recurrence relation for $v_{n}$ can be extended to a recurrence relation for

$$
\begin{aligned}
& \sum_{k=0}^{n}\left(\begin{array}{l}
n \\
k
\end{array}\right)\left(\begin{array}{c}
n+2 a \\
k+a
\end{array}\right)\left(\begin{array}{c}
n+2 b \\
k+b
\end{array}\right) \\
& \quad=\left(\begin{array}{c}
n+2 a \\
a
\end{array}\right)\left(\begin{array}{c}
n+2 b \\
b
\end{array}\right){ }_{3} F_{2}\left(\begin{array}{c}
-n,-n-a,-n-b \\
a+1, b+1
\end{array} ;-1\right)
\end{aligned}
$$


There is another way of pairing the terms to get a well poised series, so

$$
\begin{aligned}
& \sum_{k=0}^{n}\left(\begin{array}{l}
n \\
k
\end{array}\right)\left(\begin{array}{l}
n+c \\
k+a
\end{array}\right)\left(\begin{array}{c}
n+c \\
k+c-a
\end{array}\right) \\
& \quad=\left(\begin{array}{c}
n+c \\
a
\end{array}\right)\left(\begin{array}{l}
n+c \\
c-a
\end{array}\right)_{3} F_{2}\left(\begin{array}{c}
-n,-n-c+a,-n-a \\
c-a+1, a+1
\end{array} ;-1\right)
\end{aligned}
$$

also satisfies a three term recurrence relation.

Cusick's other sequence is well poised, but it is most easily treated as a special case of another class of well poised series, very well poised series. Whipple is also responsible for this transformation. He showed [15] that

$$
\begin{gathered}
{ }_{7} F_{6}\left(\begin{array}{c}
a, 1+a / 2, b, c, d, e, f \\
a / 2, a+1-b, a+1-c, a+1-d, a+1-e, a+1-f
\end{array}\right) \\
=\frac{\Gamma(a+1-d) \Gamma(a+1-e) \Gamma(a+1-f) \Gamma(a+1-d-e-f)}{\Gamma(a+1) \Gamma(a+1-d-e) \Gamma(a+1-d-f) \Gamma(a+1-e-f)} \\
{ }_{4} F_{3}(a+1-b-c, d, e, f ; a+1-b, a+1-c, d+e+f-a ; 1)
\end{gathered}
$$

when the ${ }_{4} F_{3}$ terminates and the ${ }_{7} F_{6}$ series converges. The ${ }_{7} F_{6}$ is well poised, but there is an added restriction. The factors

$$
\frac{(a / 2+1)_{n}}{(a / 2)_{n}}=\frac{(2 n+a)}{a}
$$

introduce a linear factor in the series. This can be removed by taking $f=a / 2$ or $b=a / 2$. When this is done, say with $f=a / 2$, the resulting identity is

$$
\begin{aligned}
{ }_{5} F_{4}\left(\begin{array}{c}
a, b, c, d, e \\
a+1-b, a+1-c, a+1-d, a+1-e
\end{array} ; 1\right) \\
=\frac{\Gamma(a+1-d) \Gamma(a+1-e) \Gamma(a / 2+1) \Gamma(a / 2+1-d-e)}{\Gamma(a+1) \Gamma(a+1-d-e) \Gamma(a / 2+1-d) \Gamma(a / 2+1-e)} \\
\quad{ }_{4} F_{3}\left(\begin{array}{c}
a+1-b-c, d, e, a / 2 \\
a+1-b, a+1-c, d+e-a / 2
\end{array} ; 1\right) .
\end{aligned}
$$

The ${ }_{4} F_{3}$ is balanced, but shifting $a$ by one does not lead to a series whose parameters have been changed by one, because of the $a / 2$. However take $e=(a+1) / 2$ to get

$$
\begin{aligned}
{ }_{4} F_{3}\left(\begin{array}{c}
a, b, c, d \\
a+1-b, a+1-c, a+1-d
\end{array} ;\right) \\
=\frac{\Gamma(a+1-d) \Gamma((a+1) / 2) \Gamma(a / 2+1) \Gamma(1 / 2-d)}{\Gamma(a+1) \Gamma((a+1) / 2-d) \Gamma(a / 2+1-d) \Gamma(1 / 2)} \\
{ }_{4} F_{3}\left(\begin{array}{c}
a+1-b-c, b, a / 2,(a+1) / 2 \\
a+1-b, a+1-c, d+1 / 2
\end{array} ; 1\right),
\end{aligned}
$$


and now shifting any of the parameters by one leads to a series whose parameters have been shifted by one. Thus we can find the three term recurrence relation satisfied by

$$
\begin{aligned}
& \sum_{k=0}^{n}\left(\begin{array}{l}
n \\
k
\end{array}\right)\left(\begin{array}{c}
n+2 a \\
k+a
\end{array}\right)\left(\begin{array}{c}
n+2 b \\
k+b
\end{array}\right)\left(\begin{array}{c}
n+2 c \\
k+c
\end{array}\right) \\
& \quad=\left(\begin{array}{c}
n+2 a \\
a
\end{array}\right)\left(\begin{array}{c}
n+2 b \\
b
\end{array}\right)\left(\begin{array}{c}
n+2 c \\
c
\end{array}\right)_{4} F_{3}\left(\begin{array}{c}
-n, n-a,-n-b,-n-c \\
a+1, b+1, c+1
\end{array} ; 1\right) .
\end{aligned}
$$

This is not the end of three term recurrence relations. Wilson [18] showed that very well poised two balanced (sum of top +2 equals the sum of bottom) ${ }_{9} F_{8}$ 's satisfy three-term contiguity relations, where the definition of contiguous requires that both very well poised and two balanced be preserved. There are also basic hypergeometric series extensions of all these results. A series $\Sigma a_{k}$ is a basic hypergeometric series if $a_{k+1} / a_{k}$ is a rational function of $q^{k}$ for a fixed number $q$. Only one of the balanced ${ }_{4} \varphi_{3}$ recurrence relations has been published so far. This is one that comes from a set of orthogonal polynomials [2]. The rest have been worked out by one of us (J.A.W.) and will be published in due course.

We were motivated to write this paper because of a note by Rieger [12]. He found four sequences that satisfy three term recurrence relations and derived the relations. The first two are special cases of the recurrence relation for Jacobi polynomials $[13,(4.5 .1)]$. These are the polynomials defined in (11). The other two are special cases of the recurrence relation for $h_{n}$ defined in (26).

The main interest in many of these recurrence relations is because of orthogonal polynomials. If $p_{n}(x)$ is a polynomial of degree $n,\left\{p_{n}(x)\right\}_{n=0}^{\infty}$ is a set of orthogonal polynomials if

$$
\int_{-\infty}^{\infty} p_{n}(x) p_{m}(x) d \alpha(x)=0, \quad m \neq n,
$$

for a positive measure $d \alpha(x)$. Such a polynomial set satisfies a three term recurrence relation

$$
x p_{n}(x)=A_{n} p_{n+1}(x)+B_{n} p_{n}(x)+C_{n} p_{n-1}(x)
$$

with $A_{n}, B_{n}, C_{n}$ real and $A_{n} C_{n+1}>0, n=0,1, \ldots$ Conversely a set of polynomials that satisfies (38) with the appropriate positivity conditions is a set of orthogonal polynomials. See Szegö $\left[13\right.$, Theorem 3.2.1]. At the ${ }_{4} F_{3}$ level Wilson $[17,19]$ has shown that

$$
p_{n}\left(x^{2}\right)={ }_{4} F_{3}\left(\begin{array}{c}
-n, n+a+b+c+d-1, a+i x, a-i x ; 1 \\
a+b, a+c, a+d
\end{array}\right)
$$


satisfies

$$
\int_{0}^{\infty} p_{n}\left(x^{2}\right) p_{m}\left(x^{2}\right)\left|\frac{\Gamma(a+i x) \Gamma(b+i x) \Gamma(c+i x) \Gamma(d+i x)}{\Gamma(2 i x)}\right|^{2} d x=0,
$$

$m \neq n$, when $a, b, c, d>0$. He also found their recurrence relation. It was the existence of this relation that led to the complete list given in [18].

There are other applications of these recurrence relations. See [1] for an application of a three term recurrence relation for $\mathrm{a}_{3} F_{2}$ with $x=1$, and [3] for an application of a three term recurrence relation for what at the time was thought to be a messy double sum, but which in reality can be transformed into a two balanced very well poised ${ }_{9} F_{8}$. See [9].

\section{References}

[1] R. Askey and G. Gasper, 'Jacobi polynomial expansions of Jacobi polynomials with non-negative coefficients,' Proc. Cambridge Philos. Soc. 70 (1971), 243-255.

[2] R. Askey and J. Wilson, 'A set of orthogonal polynomials that generalize the Racah coefficients or $6-j$ symbols,' SIAM J. Math. Anal. 10 (1979), 1008-1016.

[3] G. Gasper, 'Linearization of the product of Jacobi polynomials, II,' Canad. J. Math. 22 (1970), $582-593$.

[4] C. F. Gauss, 'Disquisitiones generales circa seriem infinitam..., Comment Gotting. 2 (1812), 1-46; Werke, III (1868), 123-162.

[5] E. E. Kummer, 'Über die hypergeometrische Reihe,' J. für Math. 15 (1836), 39-83, 127-172; Collected Papers, II, 75-166.

[6] M. Mendes-France, Roger Apéry et l'irrationnel, (Le Recherche, No. 97).

[7] J. F. Pfaff, 'Observations analyticae ad L. Euler's Institutiones Calculi Integralis,' vol. IV, Supplem. II et IV, Historte de 1793, Nova acta academiae scientiarum Petropolitanae, XI, 1797, 38-57. (Note, the history section is paged separately from the scientific section of this journal.)

[8] E. D. Rainville, Special functions (Macmillan, New York, 1960).

[9] M. Rahman, 'A non-negative representation of the linearization coefficients of the product of Jacobi polynomials,' Canad. J. Math. 33 (1981), 915-928.

[10] J. Raynal, 'On the definition and properties of generalized 3-j symbols,' J. Math. Phys. 19 (1978), 467-476.

[11] J. Raynal, 'On the definition annd properties of generalized $6-j$ symbols,' J. Math. Phys. 20 (1979), 2398-2415.

[12] G. J. Rieger, 'Einige Rekursionsformeln für Summen mit Binomialkoeffizienten,' $A b h$. Braunschweig. Wiss. Gesellsch 31 (1980), 137-143.

[13] G. Szegö, Orthogonal polynomials, fourth edition (Amer. Math. Soc. Colloq. Publ. 23, Amer. Math. Soc., Providence, R.I., 1975).

[14] A. van der Poorten, 'A proof that Euler missed... Apéry's proof of the irrationality of $\zeta(3)$,' Math. Intelligencer 1 (1979), 195-203.

[15] F. J. W. Whipple, 'On well-poised series, generalized hypergeometric series having parameters in pairs, each pair with the same sum,' Proc. London Math. Soc. (2) 24 (1926), 247-263.

[16] F. J. W. Whipple, 'Some transformations of generalized hypergeometric series,' Proc. London Math. Soc. (2) 26 (1927), 257-272.

[17] J. A. Wilson, 'Three-term contiguous relations and some new orthogonal polynomials,' Padé and rational approximation, edited by E. B. Saff and R. S. Varga (Academic Press, New York, 1977, 227-232). 
[18] J. A. Wilson, Hypergeometric series recurrence relations and some new orthogonal functions, (Ph.D. thesis, Univ. of Wisconsin-Madison, Madison, 1978).

[19] J. A. Wilson,'Some hypergeometric orthogonal polynomials,' SIAM J. Math. Anal. 11 (1980), 690-701.

Department of Mathematics

University of Wisconsin-Madison

Van Vleck Hall

Department of Mathematics

Madison, Wisconsin 53706

Iowa State University

Ames, Iowa 50011

U.S.A.

U.S.A. 